\title{
Germline proliferation and its control ${ }^{*}$
}

Judith Kimble ${ }^{\S}$, Sarah L. Crittenden, Howard Hughes Medical Institute, Department of Biochemistry, University of Wisconsin, Madison, WI 53706 USA

\section{Table of Contents}

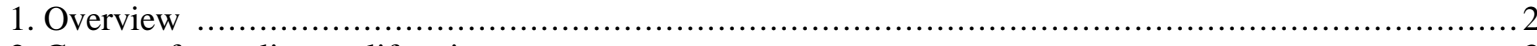

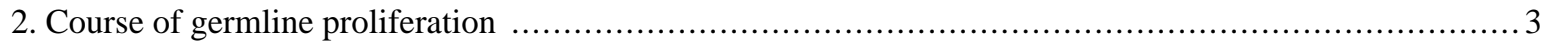

3. Control of germline proliferation by Notch signaling and the somatic gonad ..............................

3.1. The DTC and Notch signaling promote germline stem cells ....................................4

3.2. Control of germline proliferation by other cells in the developing gonad ..........................5

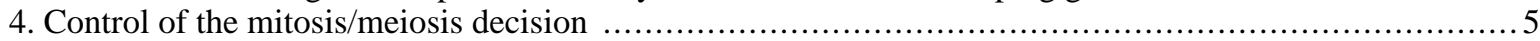

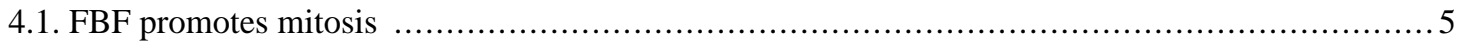

4.2. GLD-1, GLD-2, GLD-3 and NOS-3 control entry into meiosis .................................. 6

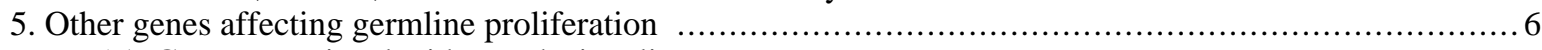

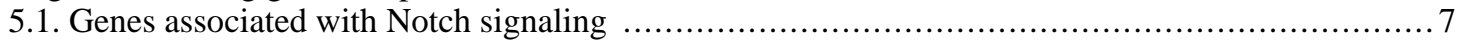

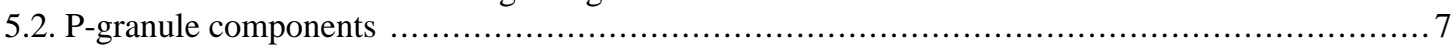

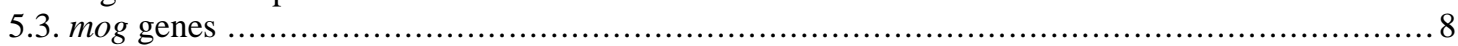

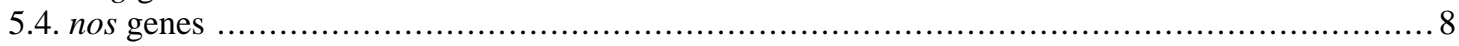

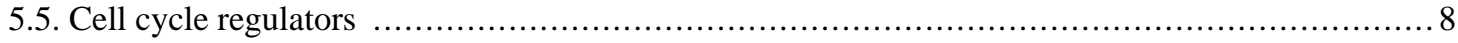

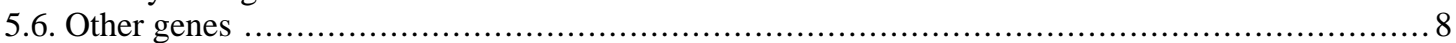

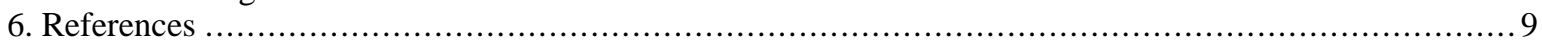

\begin{abstract}
The $C$. elegans germ line proliferates from one primordial germ cell (PGC) set aside in the early embryo to over a thousand cells in the adult. Most germline proliferation is controlled by the somatic distal tip cell, which provides a stem cell niche at the distal end of the adult gonad. The distal tip cell signals to the germ line via the Notch signaling pathway, which in turn controls a network of RNA regulators. The FBF-1 and FBF-2 RNA-binding proteins promote continued mitoses in germ cells located close to the distal tip cell, while the GLD-1, GLD-2, GLD-3, and NOS-3 RNA regulators promote entry into meiosis as germ cells leave the stem cell niche. In addition to these key regulators, many other genes affect germline proliferation.
\end{abstract}

\footnotetext{
*Edited by Susan Strome. Last revised July 25, 2005. Published August 15, 2005. This chapter should be cited as: Kimble, J. and Crittenden, S.L. Germline proliferation and its control (August 15, 2005), WormBook, ed. The C. elegans Research Community, WormBook, doi/10.1895/ wormbook.1.13.1, http://www.wormbook.org.

Copyright: (C) 2005 Judith Kimble and Sarah L. Crittenden. This is an open-access article distributed under the terms of the Creative Commons Attribution License, which permits unrestricted use, distribution, and reproduction in any medium, provided the original author and source are credited.

${ }^{\S}$ To whom correspondence should be addressed. E-mail: jekimble@wisc.edu
} 


\section{Overview}

The $C$. elegans germ line proliferates from one primordial germ cell (PGC) set aside in the early embryo to over a thousand cells in the adult. Early PGCs are incorporated into the gonadal primordium during embryogenesis, and only begin active proliferation after hatching. Continued germline mitoses take place in a somatic gonadal 'niche', which is both necessary and sufficient for proliferation; once germ cells have left this niche, they embark on the path to differentiation (entry into meiosis, progression through meiosis and gametogenesis). The location of the niche creates polarity within the maturing germ line, such that mitotic cells reside at one end (the distal end) and meiotic cells are more proximal. This chapter reviews the course of germline proliferation, its control by Notch signaling and a molecular network of RNA regulators, and ends with a brief summary of additional genes that affect germline proliferation. Two figures are provided to help visualize key aspects of germline proliferation. Figure 1 summarizes germline proliferation graphically in wild-type animals and selected mutants defective in germline proliferation. Figure 2 shows mitotic germline cells near the somatic stem cell niche and meiotic cells extending proximally away from that niche; it also provides a rough idea of where key regulators are expressed in the germ line and a simplified diagram of the regulatory circuit controlling the mitosis/meiosis decision.

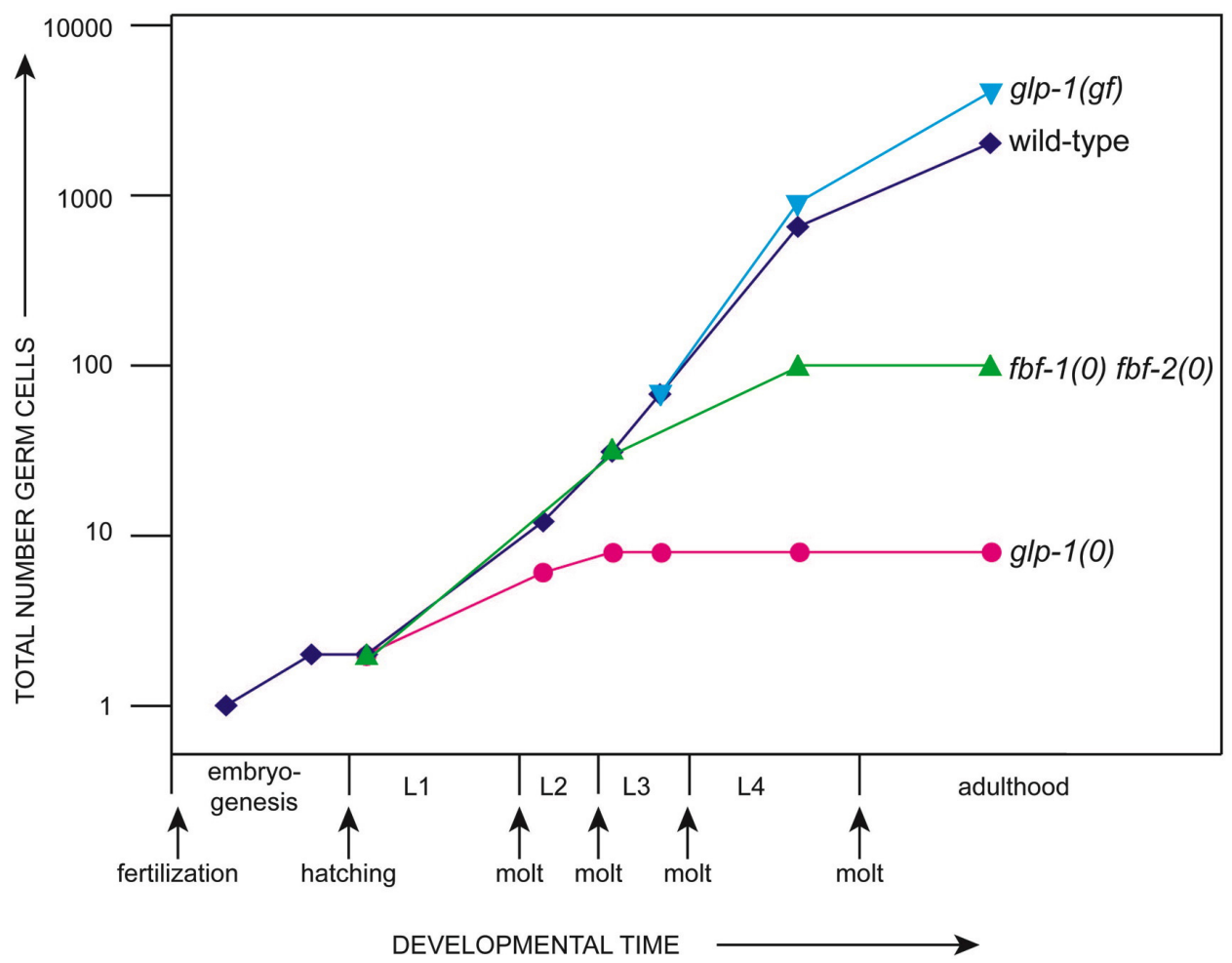

Figure 1. Proliferation in wild-type and selected mutant germ lines. The wild-type germ line begins in the early embryo with a single primordial germ cell (PGC), which divides once during embryogenesis. After hatching, the two PGCs begin their post-embryonic divisions to generate about 2000 germ cells in the adult (Kimble and White, 1981). By contrast, in $g l p-1$ null mutants, which lack the GLP-1/Notch receptor, the two PGCs make only 4-8 germ cells (Austin and Kimble, 1987); in glp-1 gain-of-function mutants, which have unregulated GLP-1/Notch receptors, they generate up to 4000 germ cells (Berry et al., 1997); and in $f b f-1(o k 91) f b f 2(q 704)$ mutants, which lack the FBF RNA-binding protein, germ cells divide until early L4 to make $~ 100$ germ cells (Crittenden et al., 2002). In both $g l p-1(0)$ and $f b f-1 f b f-2$ mutants, all germ cells leave the mitotic cell cycle, enter the meiotic cell cycle and undergo spermatogenesis. In $g l p-1(g f)$ mutants, all germ cells remain in the mitotic cell cycle. 
A.
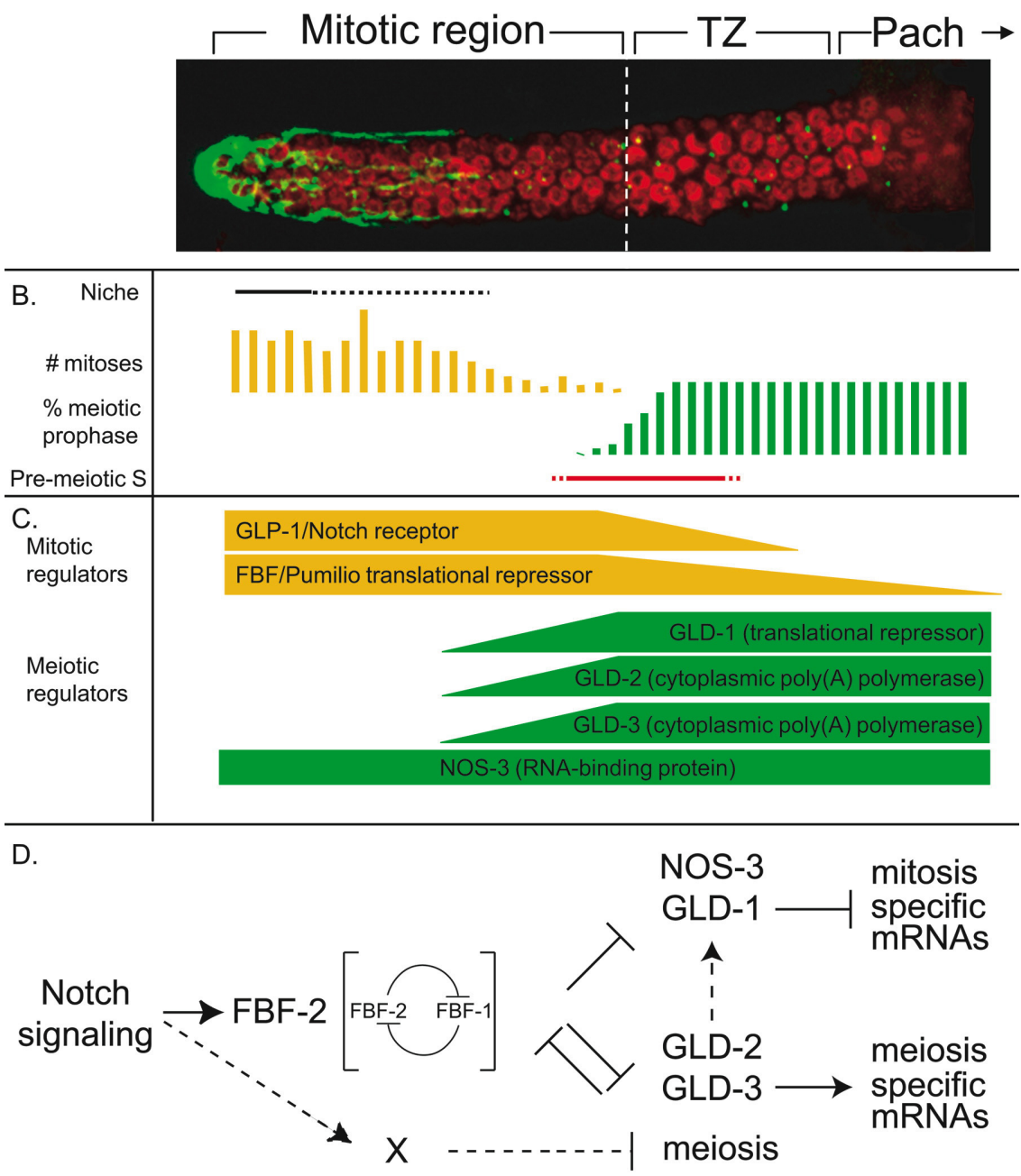

Figure 2. Regulation of the mitosis/meiosis decision in the adult germ line. (A) Top, distal end of an adult hermaphrodite gonad, dissected from the animal. Projection of confocal z series. Green DTC projection includes entire z-series, red ToPro-3 projection includes a subset of sections so nuclear morphology is clearer. Mitotic region, transition zone (TZ) and pachytene region (Pach) are marked with brackets. The DTC and its processes are visualized using GFP (green), produced under control of the lag-2 promoter; germline nuclei are visualized with ToPro-3 (red). The distal to proximal axis of the germ line extends from the DTC at the distal end to mature gametes at the proximal end. (B) Extents of the niche, mitotic region and transition zone in one stage of the hermaphrodite adult gonad. The niche (DTC and its processes) extends along the distal-proximal axis (see processes in A). The mitotic region extends $\sim 20$ cell diameters and is defined by the region in which mitotic nuclei are observed when averaged over multiple germlines. The yellow bar graph depicts relative number of phosphohistone $\mathrm{H} 3$-positive mitotic nuclei at each position in the mitotic region. The TZ begins where $60 \%$ nuclei have the crescent-shaped morphology typical of early meiotic prophase (see red crescents above). The green bar graph shows percentage of nuclei in meiotic prophase (leptotene, zygotene or pachytene) at a given position along the distal-proximal axis. Red bar indicates approximate boundary of premeiotic S-phase (SLC and JK, unpub.). This region is likely similar to the meiotic entry region described in Hansen et al. (2004). (C) Distribution of mitotic and meiotic regulators within distal germ line. Levels of GLP-1 and FBF mitotic regulators are high throughout the mitotic region and decrease as germ cells enter meiosis (Crittenden et al., 2002; Crittenden et al., 1994; Lamont et al., 2004). Conversely, levels of most meiotic regulators (GLD-1, GLD-2 and GLD-3) gradually increase in the proximal part of the mitotic region and reach high levels as germ cells enter meiotic prophase (Eckmann et al., 2002; Jones et al., 1996; Wang et al., 2002). One exception is NOS-3, which is distributed uniformly throughout the germ line (Kraemer et al., 1999). The

balance of mitotic and meiotic regulators is likely to determine whether a cell remains in mitosis or enters meiosis (for review see Crittenden et al., 2003). The sizes of the bars representing the regulators are not quantitative; for example, levels of GLD-2 and GLD-3 appear to be lower and increase less sharply than levels of GLD-1 (Eckmann et al., 2004; Eckmann et al., 2002; Jones and Schedl, 1995; Wang et al., 2002). (D) Summary of the genetic and molecular network controlling mitosis/meiosis decision. The diagrammed network is simplified, and additional regulators are certain to exist. Briefly, GLP-1 activates $f b f-2$ (along with one or more other unidentified targets (X)). FBF-1 and FBF-2 negatively regulate each other, a feedback loop critical for specifying size of the mitotic region. FBF-1 and FBF-2 also negatively regulate levels of GLD-1 and GLD-3, inhibiting meiosis in the distal region of the germ line. See text for details and references.

\section{Course of germline proliferation}

The germ line is established in the early embryo with birth of $\mathrm{P}_{4}$, the first primordial germ cell (PGC). After moving into the embryo during early gastrulation, $\mathrm{P}$ divides to generate two PGCs, called Z2 and Z3, which do not divide further until after hatching (Sulston et al., 1983). About halfway through the first larval stage, Z2 and Z3 
begin to divide; these postembryonic divisions only begin when the nutritional environment is favorable. When hatched in buffer, all postembryonic blast cells arrest, including the PGCs. Once they begin, postembryonic germline divisions are not oriented in a reproducible manner and placement of daughter cells is variable (Kimble and Hirsh, 1979). The number of germ cells increases exponentially for the first two larval stages (Figure 1); then, during the third larval stage, the most proximal germline nuclei enter meiosis, which removes them from the pool of actively dividing cells and establishes polarity in the germline. Thereafter, mitotic divisions are limited to the distal germ line, and meiotic prophase to more proximal regions. Germline proliferation arrests if larvae enter the dauer stage of development and resumes when dauers reenter the normal life cycle. The PGCs are cellular in the first larval stage, but the proliferating germ line becomes syncytial by the second larval stage. The "cells" within the germline syncytium do not divide synchronously at any time during the course of germline proliferation and therefore appear to behave autonomously; that autonomy may reflect the partial enclosure of each nucleus and its surrounding cytoplasm by a plasma membrane (Hirsh et al., 1976). The course of proliferation is similar in the two sexes, but hermaphrodites produce a total of $\sim 2000$ germline descendants partitioned into two gonadal arms, and males produce $\sim 1000$ germline descendants in a single arm (Kimble and White, 1981).

The adult germ line possesses a "mitotic region" at its distal end, and a "transition zone" more proximally (Figure 2A). Germ cells in the mitotic region serve as stem cells that both self-renew and produce differentiating gametes. In young adults (24 hours past L4), the mitotic region is composed of $\sim 225-250$ germ cells, and it extends about 20 germ cell diameters along the distal-proximal axis (Crittenden et al., 1994; Eckmann et al., 2004; Hansen et al., 2004; Lamont et al., 2004). The transition zone is the region where germline nuclei make the transition into early stages of meiotic prophase: chromosomes begin to pair and nuclear chromatin assumes a crescent-shaped morphology (Dernburg et al., 1998; Francis et al., 1995). The mechanism by which germ cells are controlled to continue mitoses in the distal region and enter meiosis as they move into the transition zone is summarized in the next two sections.

\section{Control of germline proliferation by Notch signaling and the somatic gonad}

\subsection{The DTC and Notch signaling promote germline stem cells}

The distal tip cells (DTCs) control germline proliferation during larval development and control germline mitoses in the adult. Removal of DTCs by laser ablation causes germ cells to leave the mitotic cell cycle and enter meiosis (Kimble and White, 1981), and duplication or movement of DTCs promotes germline stem cells in the new DTC location (Feng et al., 1999; Kimble and White, 1981). Therefore, DTCs are both necessary and sufficient for germline proliferation during larval development and for maintenance of germline mitoses in the adult. The germ cells located in the adult mitotic region replenish themselves and also continuously generate differentiated gametes. Therefore, they satisfy the two essential criteria for stem cells (self-renewal and generation of differentiated progeny; Watt and Hogan, 2000). The DTC serves as the stem cell niche for these adult germline stem cells.

Notch signaling controls germline proliferation during larval development and maintains germline stem cells in the adult (Crittenden et al., 2003; Kimble and Simpson, 1997; Seydoux and Schedl, 2001; see Notch signaling in the C. elegans embryo). The core components of the Notch signaling pathway include a signaling ligand (LAG-2), receptor (GLP-1), and pathway-dedicated transcription factors (LAG-1 and LAG-3/SEL-8). Upon depletion of any of these core pathway components, germ cells leave the mitotic cell cycle and enter meiosis (Austin and Kimble, 1987; Doyle et al., 2000; Lambie and Kimble, 1991; Petcherski and Kimble, 2000). By contrast, unregulated LAG-2 ligand or GLP-1 receptor leads to unregulated germline mitoses and generation of a germline tumor (Berry et al., 1997; Fitzgerald and Greenwald, 1995; Henderson et al., 1997; Pepper et al., 2003). The use of Notch signaling to promote stem cell proliferation appears to be conserved (e.g., Calvi et al., 2003; Gaiano and Fishell, 2002).

Localized Notch signaling controls the distal location of germline mitoses. This localized signaling is accomplished by expression of the signaling ligand, LAG-2, in the DTC (Henderson et al., 1994). Furthermore, ligand activity is tethered by the LAG-2 transmembrane domain - if the LAG-2 extracellular domain is expressed without its transmembrane domain, ectopic germline mitoses ensue (Fitzgerald and Greenwald, 1995; Henderson et al., 1997). In contrast to LAG-2, the GLP-1 receptor is expressed in the germ line, where it receives the LAG-2 signal and promotes mitosis at the expense of meiosis (Austin and Kimble, 1987; Crittenden et al., 1994). glp-1 mRNA is present throughout the germ line, but $g l p-1$ protein is restricted to the mitotic region (Crittenden et al., 1994). This restriction is accomplished, in part, by GLD- 1 translational repression of $g l p-1$ mRNA as germ cells enter meiosis. Consistent with this idea, GLD-1 binds directly to the $g l p-1$ 3'UTR and translationally represses $g l p-1$ mRNA in the embryo and meiotic region of the germ line (Marin and Evans, 2003; see RNA-binding proteins and Translational control of maternal RNAs). In addition, $g l p-1$ protein is high in proximal regions of germ lines with 
reduced gld-1 (Crittenden et al., 1994; Marin and Evans, 2003). The importance of GLD-1 repression to the mitosis/meiosis switch remains in question, however, because germ cells can enter meiosis in $g l d-1(0)$ mutants, despite high levels of $g l p-1$ protein (discussed in Hansen et al., 2004; Marin and Evans, 2003).

\subsection{Control of germline proliferation by other cells in the developing gonad}

Proliferation in the early larval germ line is controlled not only by the DTCs but also by two AC/VU precursor cells (Pepper et al., 2003). These AC/VU precursors make LAG-2 (Wilkinson et al., 1994) and promote germline proliferation by Notch signaling (Pepper et al., 2003). At the transition from L2 to L3, the somatic gonadal cells are rearranged to generate the somatic gonadal primordium, and one of the two AC/VU precursors adopts the anchor cell (AC) fate (Kimble and Hirsh, 1979). The AC continues to express LAG-2 (Wilkinson et al., 1994), but in normal gonads, it does not contact germ cells (Kimble and Hirsh, 1979; Seydoux et al., 1990). However, if intervening somatic gonadal cells are removed, the AC can promote germline proliferation (Seydoux et al., 1990).

The sheath/spermathecal (SS) precursor cells or their descendants are required for robust germline proliferation (McCarter et al., 1997). Removal of the SS precursors can reduce the number of adult germ cells by as much as $80 \%$, yet a mitotic region persists in SS-ablated gonads. The pro- 1 gene has recently been implicated in SS control of germline development (Killian and Hubbard, 2004).

\section{Control of the mitosis/meiosis decision}

The decision between continued mitotic division and entry into the meiotic cell cycle is controlled, at least in part, by a network of RNA regulatory proteins (Figure 2). Figure 2D diagrams a simplified view of the logic of this network. Briefly, the FBF RNA-binding protein represses mRNAs encoding regulators in each of two downstream branches of control, and Notch signaling has been linked to the RNA regulatory network by transcriptional regulation of $f b f-2$. This simplified view is certainly not complete: Notch signaling must control other regulators than FBF, and the RNA regulatory network cannot fully control the mitosis/meiosis decision. These unknowns are represented by "X" in Figure 2D. Although other regulators have been identified, their roles in the pathway have not been established to date. It should be noted that many of the regulators that control the mitosis/meiosis decision also control the sperm/oocyte decision (see Sex-determination in the germ line). Therefore, growth regulation is tightly coupled to the control of sex determination by use of the same regulators in both processes.

\subsection{FBF promotes mitosis}

Two nearly identical proteins, called FBF-1 and FBF-2, are required for continued mitotic divisions in late larval and adult animals, including maintenance of adult germline stem cells (Crittenden et al., 2002). In $f b f-1 f b f-2$ double mutants, germline proliferation ceases during the fourth larval stage, and all germ cells enter meiosis and differentiate into sperm. Therefore, FBF is a key regulator of adult germline stem cells.

FBF-1 and FBF-2 are sequence-specific RNA binding proteins, and their binding activities are indistinguishable in vitro (Bernstein et al., 2005; Zhang et al., 1997). Collectively FBF-1 and FBF-2 are called FBF. FBF belongs to the Puf protein family (for Pumilio and FBF; Wickens et al., 2002; Zhang et al., 1997; link to RNA binding proteins). FBF binds regulatory elements in the 3' untranslated regions (3'UTRs) of target mRNAs, and represses target mRNAs as do its homologs in yeast and Drosophila (Wickens et al., 2002).

Several FBF target mRNAs are known. Two targets are the gld-1 and gld-3 mRNAs (Crittenden et al., 2002; Eckmann et al., 2004), both encoding regulators that promote meiosis (see below). Two other targets are the $f b f-1$ and $f b f-2$ mRNAs themselves. This FBF autoregulation maintains FBF levels at a relatively low level in the wild-type germ line (Lamont et al., 2004).

FBF-2 appears to be a direct downstream target of Notch signaling (Lamont et al., 2004). Consistent with this idea, $f b f-2$ mRNA and FBF-2 protein are localized to the distal-most germ line, FBF-2 expression is responsive to Notch signaling, and the 5 ' flanking region of the $f b f-2$ gene possesses four LAG- 1 binding sites. However, this direct link between Notch signaling and $f b f-2$ expression is not sufficient to explain Notch control of germline proliferation, and additional Notch target genes must exist.

FBF-1 and FBF-2 have distinct effects on the size of the mitotic region (Lamont et al., 2004). Wild-type germ lines possess 225-250 cells in the mitotic region (Eckmann et al., 2004; Hansen et al., 2004; Lamont et al., 2004). By contrast, germ lines lacking $f b f-1$ activity have smaller mitotic regions with only 200 cells, and germ lines 
lacking $f b f-2$ activity have larger mitotic regions with $\sim 400$ cells. This difference can be explained, at least in part, by the differential regulation of the two $f b f$ genes. Thus, the size of the mitotic region is determined by FBF- 2 in $f b f-1$ single mutants, and FBF-2 is spatially limited to the distal-most region. By contrast, the size of the mitotic region is determined by $f b f-1$ in $f b f-2$ single mutants, where the distribution of $f b f-1$ expands. Therefore, these duplicated genes have distinct roles in controlling the number of cells within the mitotic region.

\subsection{GLD-1, GLD-2, GLD-3 and NOS-3 control entry into meiosis}

Four key regulators control entry into meiosis: GLD-1, GLD-2, GLD-3 (for germ line development) and NOS-3 (for Nanos; Eckmann et al., 2004; Hansen et al., 2004; Hansen et al., 2004; Kadyk and Kimble, 1998). GLD-1, GLD-2, GLD-3 and NOS-3 are all RNA regulators that control multiple steps in germline development. Here, we briefly summarize their molecular identities and focus on how each fits into the regulatory network controlling germline proliferation.

The RNA regulators that promote entry into meiosis fall into two major branches of regulation: GLD-1 and NOS-3 act together in one branch, while GLD-2 and GLD-3 act together in the other branch (Figure 2B). Null mutants for any one of these key regulators enter meiosis normally, but double mutants that remove activities from both branches virtually abolish entry into meiosis. For example, gld-l single mutant germ lines enter meiosis normally (Francis et al., 1995), but gld-1 gld-2 double mutants do not (Kadyk and Kimble, 1998). Hansen et al. (2004) have recently noted that a few germ cells can enter meiosis in animals lacking activities from both branches, suggesting the existence of an additional minor branch that can promote entry into meiosis (Figure 1D).

- GLD-1 belongs to the STAR/KH family of RNA-binding proteins (Jones and Schedl, 1995); it is a sequence-specific RNA-binding protein (Ryder et al., 2004) and functions as a translational repressor (Jan et al., 1999; Lee et al., 2001). GLD-1 is likely to repress the activity of mRNAs that encode proteins critical for germline mitoses. Consistent with this idea, GLD-1 represses the activity of $g l p-1$ mRNA, which encodes the glp-1/Notch receptor (Marin and Evans, 2003). Other GLD-1 targets relevant to the mitosis/meiosis decision have not been identified.

- NOS-3 belongs to the Nanos family of zinc finger proteins and has been implicated in translational repression (Kraemer et al., 1999). The mechanism of NOS-3 control over entry into meiosis appears to involve activation of GLD-1 (Hansen et al., 2004); that activation might involve repression of a repressor or a novel molecular mechanism for NOS-3.

- GLD-2 and GLD-3 function together to promote entry into meiosis (Eckmann et al., 2004). The GLD-2 and GLD-3 proteins bind to each other in vitro, and co-immunoprecipitate with each other from worm extracts. GLD-2 is the catalytic subunit of a cytoplasmic poly(A) polymerase (PAP; Wang et al., 2002); its targets are not known. GLD-3 is a homolog of the Bicaudal-C RNA-binding protein; it possesses five KH motifs and promotes GLD-2 PAP activity (Eckmann et al., 2002; Wang et al., 2002). The GLD-2/GLD-3 poly(A) polymerase has been proposed to promote entry into meiosis by activating the activity of meiosis-promoting mRNAs.

GLD-1, GLD-2 and NOS-3 all act downstream of FBF genetically, and gld-1 mRNA is a direct target of FBF repression (Crittenden et al., 2002; Eckmann et al., 2004; Hansen et al., 2004). GLD-3 acts largely downstream of FBF to promote meiosis, and gld-3 mRNA also appears to be a direct target of FBF repression (Eckmann et al., 2004). However, GLD-3 protein interferes with FBF binding activity (Eckmann et al., 2002), and genetic analyses suggest that GLD-3 may feedback negatively on FBF activity (Eckmann et al., 2004).

\section{Other genes affecting germline proliferation}

In this section, we briefly describe additional genes that affect normal germline proliferation. As mentioned earlier, the simplified regulatory pathway presented in Figure 2D is certainly not complete. Additional regulators are clearly required (e.g., Hansen et al., 2004; Hansen et al., 2004; Lamont et al., 2004). The "X" in Figure 2D may represent one or more additional branches of regulation that work together with the RNA regulatory network to control proliferation and the mitosis/meiosis decision. The identification of many of these genes as RNA regulatory proteins is striking. 


\subsection{Genes associated with Notch signaling}

\subsection{1. ego genes (for enhancer of $g / p-1$ ).}

Five ego genes were identified as enhancers of $g l p-1$; in addition, alleles of $l a g-1$ and $g l p-4$ were found in the same screen (Qiao et al., 1995). EGO-1 is related to RNA-directed RNA polymerase and is also critical for RNAi (Smardon et al., 2000). This finding implicates RNA interference, and perhaps micro RNAs, in control of germline proliferation, although no candidates have been found to date. An ego-l null mutant has a number of germline defects, including early meiotic progression and gametogenesis (Smardon et al., 2000).

An additional enhancer of glp-1 is epn-1, which encodes the C. elegans homolog of epsin (Tian et al., 2004). Epsin is an intregral component of the endocytotic machinery (Chen et al., 1998) and appears to be critical for generation or function of the DTC ligand (Tian et al., 2004).

\subsection{2. sog genes (uppressors of $g l p-1$ )}

Six $\operatorname{sog}$ genes were identified as suppressors of glp-1 (Maine and Kimble, 1993); these genes have no phenotype on their own and have not been pursued. Several morphogenetic mutants are also glp-l suppressors (Maine and Kimble, 1989) as well as emb-5 suppressors (Nishiwaki and Miwa, 1998). These morphogenetic suppressors encode collagens (dpy-2, dpy-3/dpy-9, dpy-10 and sqt-1), suggesting either a possible link between extracellular matrix and Notch signaling or a link between chaperones and the efficiency of Notch signaling.

\subsection{3. sel genes (suppressors and enhancers of the 느N-12/Notch receptor).}

Several sel genes also affect the $\mathrm{glp}-1 /$ Notch receptor and its control of germline proliferation (Sundaram and Greenwald, 1993; Tax et al., 1997). These are detailed in the section on signaling by LIN-12 and glp-1 receptors (see LIN-12/Notch signaling in C. elegans). Briefly, they include SEL-1, a negative regulator that may participate in receptor turnover (Grant and Greenwald, 1996; Grant and Greenwald, 1997); SEL-5, a kinase that facilitates signaling (Fares and Greenwald, 1999); SEL-7, a novel nuclear protein that promotes LIN-12 activity (Chen et al., 2004); SEL-9, a p24 ortholog implicated in receptor trafficking (Wen and Greenwald, 1999); SEL-10, an F-box protein that negatively regulates receptor activity (Hubbard et al., 1997; Oberg et al., 2001; Wu et al., 1998); SEL-12, an ortholog of human presenilin that mediates receptor cleavage (De Strooper et al., 1999; Levitan and Greenwald, 1995); and SUP-17, an ADAM metalloprotease that cleaves the receptor constitutively (Tax et al., 1997; Wen et al., 1997; see LIN-12/Notch signaling in C. elegans).

\subsection{4. emb-5 (for embryonic lethal).}

EMB-5 was identified in a yeast two-hybrid screen using LIN-12 ankyrin repeats as bait (Hubbard et al., 1996). EMB-5 protein is similar to a yeast chromatin protein, called Spt6p, which may have a role in controlling chromatin structure. EMB-5 acts downstream of GLP-1 and is required continuously for germline proliferation (Hubbard et al., 1996). The effect of emb-5 is suppressed by some glp-1 suppressors (see above section on sog genes).

\subsection{P-granule components}

Several P-granule components are critical for normal germline proliferation as well as for other aspects of germline development and early embryogenesis (see Specification of the germ line). We also note that GLD-1, GLD-2 and GLD-3 are associated with P granules (Eckmann et al., 2002; Jones et al., 1996; Wang et al., 2002), but these three RNA regulators are discussed above.

1. PGL family proteins. PGL (for $\underline{P}$ granule defective) proteins are critical for germline proliferation (Kawasaki et al., 2004; Kawasaki et al., 1998). PGL-1 and PGL-3 possess RGG-box domains and are likely to bind RNA. $p g l-1$ single mutants have underproliferated germ lines, and animals depleted for $p g l-1$ and $p g l-3$ are more severely affected.

2. GLH family proteins and their interacting partners. GLH (for germ line helicase) proteins are the C. elegans counterparts of the VASA DEAD-box helicase, and are called GLH-1 - GLH-4 (Gruidl et al., 1996; Kuznicki et al., 2000; Roussell and Bennett, 1993). Two GLH proteins are critical for germline proliferation (Kuznicki et al., 2000). In addition, two GLH-interacting proteins are also critical for normal germline proliferation: CSN-5, 
which is closely related to subunit 5 of COP9 signalosomes, and KGB-1, a putative JNK MAP kinase (Smith et al., 2002).

3. IFF family proteins. The IFF-1 (for initiation factor five) protein is the C. elegans counterpart of eukaryotic initiation factor 5A. Animals depleted for IFF-1 have an underproliferated germ line, perhaps because PGL-1 is not recruited normally to $\mathrm{P}$ granules (Hanazawa et al., 2004).

\section{3. $\operatorname{mog}$ genes}

The mog genes (for masculinization of the germ line) promote both proliferation and oogenesis (Graham and Kimble, 1993; Graham et al., 1993). Animals depleted for any of six mog genes have an underproliferated germ line. The mog-1, mog-4 and mog-5 genes encode putative DEAH-box RNA helicases that are the single C. elegans homologs of PRP16, PRP2 and PRP22 respectively (Puoti and Kimble, 1999, 2000). These PRP proteins have been implicated in splicing in yeast (Schwer and Guthrie, 1991), but do not appear to affect splicing in nematodes (Puoti and Kimble, 1999). The mog-6 gene encodes a cyclophilin homolog (Belfiore et al., 2004). These MOG proteins are all nuclear and affect a reporter transgene harboring an FBF regulated 3'UTR. Their mechanism of action remains unknown.

Double mutants that lack both one mog gene as well as gld-3 have germline tumors (Belfiore et al., 2004). Therefore, the mog genes may operate in the gld-1/nos-3 branch described above or may define a separate regulatory branch.

\section{4. nos genes}

Nanos (NOS) proteins are critical for several aspects of germline development: proliferation (this section), sex determination (see Sex-determination in the germ line), germline survival and cell death (see Germline survival and cell death). NOS-3 is a key regulator required for entry into meiosis and is discussed above. In addition, NOS-1 and NOS-2 have important effects on germline proliferation and survival (Kraemer et al., 1999; Subramaniam and Seydoux, 1999). Specifically, in wild-type animals, NOS-1 and NOS-2 promote mitotic arrest in the early germ line: $\mathrm{Z} 2$ and Z3 divide prematurely in animals depleted of nos activity. Furthermore, later in larval development, the three nos genes have overlapping functions required for normal germline proliferation and survival.

\subsection{Cell cycle regulators}

The basic cell cycle machinery controls virtually all somatic cell divisions, and hence controls generation of somatic gonadal cells that promote germline proliferation; these genes may also have roles within the germ line. Cell cycle regulators that appear to function in the germ line include: NCC-1/CDK-1 (Boxem et al., 1999), CYE-1 (Brodigan et al., 2003; Fay and Han, 2000), CUL-2 and CUL-4 (Feng et al., 1999; Zhong et al., 2003), CDC25.1 (Ashcroft and Golden, 2002), and APC/C subunits: EMB-30 (Furuta et al., 2000), EMB-27, MAT-1, MAT-2 and MAT-3 (Golden et al., 2000; Shakes et al., 2003; see Cell cycle regulation)

\subsection{Other genes}

- $g l p-3 / e f t-3$ and $g l p-4$. The $g l p-3$ and $g l p-4$ genes are both critical for germline proliferation (Beanan and Strome, 1992; Kadyk and Kimble, 1998; Maciejowski et al., 2005). In $g l p-3$ and $g l p-4$ mutants, germ cell mitotic divisions are severely reduced, but continue into adulthood (Beanan and Strome, 1992; Kadyk et al., 1997). The only one of these genes cloned to date is $g l p-3$, which encodes a homolog of elongation factor 1- $\alpha$ (Maciejowski et al., 2005).

- $\quad p a b-1$ and $r p l-11.1$. Two genes required for early germline proliferation include $p a b-1$ and $r p l-11.1$ (Ciosk et al., 2004; Maciejowski et al., 2005). The pab-1 gene encodes one of two poly(A) binding proteins encoded by the genome. rpl-11.1 encodes the L11 protein of the large ribosomal subunit. PAB-1 has also been linked to the control of germline proliferation by studies with ATX-2 (see below).

- ATX-2. ATX-2 is the C. elegans counterpart of mammalian atx-2, a protein that binds poly(A) binding protein (PAB; Ciosk et al., 2004; Maine et al., 2004). Depletion of ATX-2 from C. elegans reduces germ cell number and also masculinizes the germ line. One explanation of these effects is an increase in GLD-1 expression in atx-2(RNAi) animals as well as an alteration in GLD-1 activity (Ciosk et al., 2004). 
- MRG-1. MRG-1 is the C. elegans counterpart of the human mortality factor-related gene 4 product (MRG-15), which contains a chromodomain. MRG-1 is required maternally for post-embryonic proliferation of the germ line (Fujita et al., 2002; Fujita et al., 2003).

- PRG-1. prg-1(RNAi) animals have decreased germline proliferation, and PRG-1 is a PIWI-related protein (Cox et al., 1998). PIWI and its homologs are central components of the RISC complex and have been implicated in the "slicing activity" of this complex (Parker et al., 2004, and references therein; Song et al., 2004). This finding therefore complements the ego- 1 data (Smardon et al., 2000) to link controls of germline proliferation and RNA interference.

- SPK-1. SPK-1 is an SR protein kinase-related gene product. spk-1(RNAi) animals have small germ lines and few gametes (Kuroyanagi et al., 2000).

\section{References}

Ashcroft, N., and Golden, A. (2002). CDC-25.1 regulates germline proliferation in Caenorhabditis elegans. Genesis 33, 1-7. Abstract Article

Austin, J., and Kimble, J. (1987). glp-1 is required in the germ line for regulation of the decision between mitosis and meiosis in C. elegans. Cell 51, 589-599. Abstract Article

Beanan, M.J., and Strome, S. (1992). Characterization of a germ-line proliferation mutation in C. elegans. Development 116, 755-766. Abstract

Belfiore, M., Pugnale, P., Saudan, Z., and Puoti, A. (2004). Roles of the C. elegans cyclophilin-like protein MOG-6 in MEP-1 binding and germline fates. Development 131, 2935-2945. Abstract Article

Bernstein, D., Hook, B., Hajarnavis, A., Opperman, L., and Wickens, M. (2005). Binding specificity and mRNA targets of a C. elegans PUF protein, FBF-1. RNA 11, 447-458. Article

Berry, L.W., Westlund, B., and Schedl, T. (1997). Germ-line tumor formation caused by activation of glp-1, a Caenorhabditis elegans member of the Notch family of receptors. Development 124, 925-936. Abstract

Boxem, M., Srinivasan, D.G., and van den Heuvel, S. (1999). The Caenorhabditis elegans gene ncc-1 encodes a $c d c 2$-related kinase required for $\mathrm{M}$ phase in meiotic and mitotic cell divisions, but not for $\mathrm{S}$ phase. Development 126, 2227-2239. Abstract

Brodigan, T.M., Liu, J., Park, M., Kipreos, E.T., and Krause, M. (2003). Cyclin E expression during development in Caenorhabditis elegans. Dev. Biol. 254, 102-115. Abstract Article

Calvi, L.M., Adams, G.B., Weibrecht, K.W., Weber, J.M., Olson, D.P., Knight, M.C., Martin, R.P., Schipani, E., Divieti, P., Bringhurst, F.R., et al. (2003). Osteoblastic cells regulate the haematopoietic stem cell niche. Nature 425, 841-846. Abstract Article

Chen, H., Fre, S., Slepnev, V.I., Capua, M.R., Takei, K., Butler, M.H., Di Fiore, P.P., and De Camilli, P. (1998). Epsin is an EH-domain-binding protein implicated in clathrin-mediated endocytosis. Nature 394, 793-797. Abstract Article

Chen, J., Li, X., and Greenwald, I. (2004). sel-7, a positive regulator of lin-12 activity, encodes a novel nuclear protein in Caenorhabditis elegans. Genetics 166, 151-160. Abstract Article

Ciosk, R., DePalma, M., and Priess, J.R. (2004). ATX-2, the C. elegans ortholog of ataxin 2, functions in translational regulation in the germline. Development 131, 4831-4841. Abstract Article

Cox, D.N., Chao, A., Baker, J., Chang, L., Qiao, D., and Lin, H. (1998). A novel class of evolutionarily conserved genes defined by piwi are essential for stem cell self-renewal. Genes Dev. 12, 3715-3727. Abstract 
Crittenden, S.L., Bernstein, D.S., Bachorik, J.L., Thompson, B.E., Gallegos, M., Petcherski, A.G., Moulder, G., Barstead, R., Wickens, M., and Kimble, J. (2002). A conserved RNA-binding protein controls germline stem cells in Caenorhabditis elegans. Nature 417, 660-663. Abstract Article

Crittenden, S.L., Eckmann, C.R., Wang, L., Bernstein, D.S., Wickens, M., and Kimble, J. (2003). Regulation of the mitosis/meiosis decision in the Caenorhabditis elegans germline. Philos. Trans. R. Soc. Lond. B. 358, 1359-1362. Abstract Article

Crittenden, S.L., Troemel, E.R., Evans, T.C., and Kimble, J. (1994). GLP-1 is localized to the mitotic region of the C. elegans germ line. Development 120, 2901-2911. Abstract

De Strooper, B., Annaert, W., Cupers, P., Saftig, P., Craessaerts, K., Mumm, J.S., Schroeter, E.H., Schrijvers, V., Wolfe, M.S., Ray, W.J., et al. (1999). A presenilin-1-dependent $\gamma$-secretase-like protease mediates release of Notch intracellular domain. Nature 398, 518-522. Abstract Article

Dernburg, A.F., McDonald, K., Moulder, G., Barstead, R., Dresser, M., and Villeneuve, A.M. (1998). Meiotic recombination in C. elegans initiates by a conserved mechanism and is dispensable for homologous chromosome synapsis. Cell 94, 387-398. Abstract Article

Doyle, T.G., Wen, C., and Greenwald, I. (2000). SEL-8, a nuclear protein required for LIN-12 and GLP-1 signaling in Caenorhabditis elegans. Proc. Natl. Acad. Sci. USA 97, 7877-7881. Abstract Article

Eckmann, C.R., Crittenden, S.L., Suh, N., and Kimble, J. (2004). GLD-3 and control of the mitosis/meiosis decision in the germline of Caenorhabditis elegans. Genetics 168, 147-160. Abstract Article

Eckmann, C.R., Kraemer, B., Wickens, M., and Kimble, J. (2002). GLD-3, a bicaudal-C homolog that inhibits FBF to control germline sex determination in C. elegans. Dev. Cell 3, 697-710. Abstract Article

Fares, H., and Greenwald, I. (1999). SEL-5, a serine/threonine kinase that facilitates lin-12 activity in Caenorhabditis elegans. Genetics 153, 1641-1654. Abstract

Fay, D.S., and Han, M. (2000). Mutations in cye-1, a Caenorhabditis elegans cyclin E homolog, reveal coordination between cell-cycle control and vulval development. Development 127, 4049-4060. Abstract

Feng, H., Zhong, W., Punkosdy, G., Gu, S., Zhou, L., Seabolt, E.K., and Kipreos, E.T. (1999). CUL-2 is required for the G1-to-S phase transition and mitotic chromosome condensation in Caenorhabditis elegans. Nat. Cell Biol. 1, 486-492. Abstract Article

Fitzgerald, K., and Greenwald, I. (1995). Interchangeability of Caenorhabditis elegans DSL proteins and intrinsic signalling activity of their extracellular domains in vivo. Development 121, 4275-4282. Abstract

Francis, R., Barton, M.K., Kimble, J., and Schedl, T. (1995). gld-1, a tumor suppressor gene required for oocyte development in Caenorhabditis elegans. Genetics 139, 579-606. Abstract

Fujita, M., Takasaki, T., Nakajima, N., Kawano, T., Shimura, Y., and Sakamoto, H. (2002). MRG-1, a mortality factor-related chromodomain protein, is required maternally for primordial germ cells to initiate mitotic proliferation in C. elegans. Mech. Dev. 114, 61-69. Abstract Article

Fujita, M., Takasaki, T., Nakajima, N., Kawano, T., Shimura, Y., and Sakamoto, H. (2003). Corrigendum to "MRG-1, a mortality factor-related chromodomain protein, is required maternally for primordial germ cells to initiate mitotic proliferation in C. elegans": [Mech. Dev. 114 (2002) 61-69]. Mech. Dev. 120, 397. Article

Furuta, T., Tuck, S., Kirchner, J., Koch, B., Auty, R., Kitagawa, R., Rose, A.M., and Greenstein, D. (2000). EMB-30: An APC4 homologue required for metaphase-to-anaphase transitions during meiosis and mitosis in Caenorhabditis elegans. Mol. Biol. Cell 11, 1401-1419. Abstract

Gaiano, N., and Fishell, G. (2002). The role of Notch in promoting glial and neural stem cell fates. Annu. Rev. Neurosci 25, 471-490. Abstract Article 
Golden, A., Sadler, P.L., Wallenfang, M.R., Schumacher, J.M., Hamill, D.R., Bates, G., Bowerman, B., Seydoux, G., and Shakes, D.C. (2000). Metaphase to anaphase (mat) transition-defective mutants in Caenorhabditis elegans. J. Cell Biol. 151, 1469-1482. Abstract Article

Graham, P.L., and Kimble, J. (1993). The mog- 1 gene is required for the switch from spermatogenesis to oogenesis in Caenorhabditis elegans. Genetics 133, 919-931. Abstract

Graham, P.L., Schedl, T., and Kimble, J. (1993). More mog genes that influence the switch from spermatogenesis to oogenesis in the hermaphrodite germ line of Caenorhabditis elegans. Dev. Genet. 14, 471-484. Abstract Article

Grant, B., and Greenwald, I. (1996). The Caenorhabditis elegans sel-1 gene, a negative regulator of lin-12 and glp-1, encodes a predicted extracellular protein. Genetics 143, 237-247. Abstract

Grant, B., and Greenwald, I. (1997). Structure, function and expression of SEL-1, a negative regulator of LIN-12 and GLP-1 in C. elegans. Development 124, 637-644. Abstract

Gruidl, M.E., Smith, P.A., Kuznicki, K.A., McCrone, J.S., Kirchner, J., Roussell, D.L., Strome, S., and Bennett, K.L. (1996). Multiple potential germ-line helicases are components of the germ-line-specific P granules of Caenorhabditis elegans. Proc. Natl. Acad. Sci. USA 93, 13837-13842. Abstract Article

Hanazawa, M., Kawasaki, I., Kunitomo, H., Gengyo-Ando, K., Bennett, K.L., Mitani, S., and Iino, Y. (2004). The Caenorhabditis elegans eukaryotic initiation factor 5A homologue, IFF-1, is required for germ cell proliferation, gametogenesis and localization of the P-granule component PGL-1. Mech. Dev. 121, 213-224. Abstract Article

Hansen, D., Hubbard, E.J. A., and Schedl, T. (2004). Multi-pathway control of the proliferation versus meiotic development decision in the Caenorhabditis elegans germline. Dev. Biol. 268, 342-357. Abstract Article

Hansen, D., Wilson-Berry, L., Dang, T., and Schedl, T. (2004). Control of the proliferation versus meiotic development decision in the C. elegans germline through regulation of GLD-1 protein accumulation. Development 131, 93-104. Abstract Article

Henderson, S.T., Gao, D., Christensen, S., and Kimble, J. (1997). Functional domains of LAG-2, a putative signaling ligand for LIN-12 and GLP-1 receptors in Caenorhabditis elegans. Mol. Biol. Cell 8, 1751-1762. Abstract

Henderson, S.T., Gao, D., Lambie, E.J., and Kimble, J. (1994). lag-2 may encode a signaling ligand for the GLP-1 and LIN-12 receptors of C. elegans. Development 120, 2913-2924. Abstract

Hirsh, D., Oppenheim, D., and Klass, M. (1976). Development of the reproductive system of Caenorhabditis elegans. Dev. Biol. 49, 200-219. Abstract Article

Hubbard, E.J. A., Dong, Q., and Greenwald, I. (1996). Evidence for physical and functional association between EMB-5 and LIN-12 in Caenorhabditis elegans. Science 273, 112-115. Abstract

Hubbard, E.J. A., Wu, G., Kitajewski, J., and Greenwald, I. (1997). sel-10, a negative regulator of lin-12 activity in Caenorhabditis elegans, encodes a member of the CDC4 family of proteins. Genes Dev. 11, 3182-3193. Abstract

Jan, E., Motzny, C.K., Graves, L.E., and Goodwin, E.B. (1999). The STAR protein, GLD-1, is a translational regulator of sexual identity in Caenorhabditis elegans. EMBO J. 18, 258-269 Abstract Article

Jones, A.R., Francis, R., and Schedl, T. (1996). GLD-1, a cytoplasmic protein essential for oocyte differentiation, shows stage- and sex-specific expression during Caenorhabditis elegans germline development. Dev. Biol. 180, 165-183. Abstract Article

Jones, A.R., and Schedl, T. (1995). Mutations in gld-1, a female germ cell-specific tumor suppressor gene in Caenorhabditis elegans, affect a conserved domain also found in Src-associated protein Sam68. Genes Dev. 9, 1491-1504. Abstract

Kadyk, L.C., and Kimble, J. (1998). Genetic regulation of entry into meiosis in Caenorhabditis elegans. Development 125, 1803-1813. Abstract 
Kadyk, L.C., Lambie, E.J., and Kimble, J. (1997). glp-3 is required for mitosis and meiosis in the Caenorhabditis elegans germ line. Genetics 145, 111-121. Abstract

Kawasaki, I., Amiri, A., Fan, Y., Meyer, N., Dunkelbarger, S., Motohashi, T., Karashima, T., Bossinger, O., and Strome, S. (2004). The PGL family proteins associate with germ granules and function redundantly in Caenorhabditis elegans germline development. Genetics 167, 645-661. Abstract Article

Kawasaki, I., Shim, Y.-H., Kirchner, J., Kaminker, J., Wood, W.B., and Strome, S. (1998). PGL-1, a predicted RNA-binding component of germ granules, is essential for fertility in C. elegans. Cell 94, 635-645. Abstract Article

Killian, D.J., and Hubbard, E.J. A. (2004). C. elegans pro-1 activity is required for soma/germline interactions that influence proliferation and differentiation in the germ line. Development 131, 1267-1278. Abstract Article

Kimble, J., and Hirsh, D. (1979). The postembryonic cell lineages of the hermaphrodite and male gonads in Caenorhabditis elegans. Dev. Biol. 70, 396-417. Abstract Article

Kimble, J., and Simpson, P. (1997). The LIN-12/Notch signaling pathway and its regulation. Annu. Rev. Cell Dev. Biol. 13, 333-361. Abstract Article

Kimble, J.E., and White, J.G. (1981). On the control of germ cell development in Caenorhabditis elegans. Dev. Biol. 81, 208-219. Abstract Article

Kraemer, B., Crittenden, S., Gallegos, M., Moulder, G., Barstead, R., Kimble, J., and Wickens, M. (1999). NANOS-3 and FBF proteins physically interact to control the sperm-oocyte switch in Caenorhabditis elegans. Curr. Biol. 9, 1009-1018. Abstract Article

Kuroyanagi, H., Kimura, T., Wada, K., Hisamoto, N., Matsumoto, K., and Hagiwara, M. (2000). SPK-1, a $C$. elegans SR protein kinase homologue, is essential for embryogenesis and required for germline development. Mech. Dev. 99, 51-64. Abstract Article

Kuznicki, K.A., Smith, P.A., Leung-Chiu, W.M. A., Estevez, A.O., Scott, H.C., and Bennett, K.L. (2000). Combinatorial RNA interference indicates GLH-4 can compensate for GLH-1; these two P granule components are critical for fertility in C. elegans. Development 127, 2907-2916. Abstract

Lambie, E.J., and Kimble, J. (1991). Two homologous regulatory genes, lin-12 and glp-1, have overlapping functions. Development 112, 231-240. Abstract

Lamont, L.B., Crittenden, S.L., Bernstein, D., Wickens, M., and Kimble, J. (2004). FBF-1 and FBF-2 regulate the size of the mitotic region in the C. elegans germline. Dev. Cell 7, 697-707. Abstract Article

Lee, M., Cram, E.J., Shen, B., and Schwarzbauer, J.E. (2001). Roles for $\beta$ pat-3 integrins in development and function of Caenorhabditis elegans muscles and gonads. J. Biol. Chem. 276, 36404-36410. Abstract Article

Levitan, D., and Greenwald, I. (1995). Facilitation of lin-12-mediated signaling by sel-12, a Caenorhabditis elegans S182 Alzheimer's disease gene. Nature 377, 351-354. Abstract Article

Maciejowski, J., Ahn, J.H., Cipriani, P.G., Killian, D.J., Chaudhary, A.L., Lee, J.I., Voutev, R., Johnsen, R.C., Baillie, D.L., Gunsalus, K.C., et al. (2005). Autosomal genes of autosomal/X-linked duplicated gene pairs and germ-line proliferation in C. elegans. Genetics 169, 1997-2011. Abstract Article

Maine, E.M., Hansen, D., Springer, D., and Vought, V.E. (2004). Caenorhabditis elegans atx-2 promotes germline proliferation and the oocyte fate. Genetics 168, 817-830. Abstract Article

Maine, E.M., and Kimble, J. (1989). Identification of genes that interact with $g l p-1$, a gene required for inductive cell interactions in Caenorhabditis elegans. Development 105, 133-143. Abstract

Maine, E.M., and Kimble, J. (1993). Suppressors of $g l p-1$, a gene required for cell communication during development in Caenorhabditis elegans, define a set of interacting genes. Genetics 135, 1011-1022. Abstract 
Marin, V.A., and Evans, T.C. (2003). Translational repression of a C. elegans Notch mRNA by the STAR/KH domain protein GLD-1. Development 130, 2623-2632. Abstract Article

McCarter, J., Bartlett, B., Dang, T., and Schedl, T. (1997). Soma-germ cell interactions in Caenorhabditis elegans: multiple events of hermaphrodite germline development require the somatic sheath and spermathecal lineages. Dev. Biol. 181, 121-143. Abstract Article

Nishiwaki, K., and Miwa, J. (1998). Mutations in genes encoding extracellular matrix proteins suppress the emb-5 gastrulation defect in Caenorhabditis elegans. Mol. Gen. Genet. 259, 2-12. Abstract Article

Oberg, C., Li, J., Pauley, A., Wolf, E., Gurney, M., and Lendahl, U. (2001). The Notch intracellular domain is ubiquitinated and negatively regulated by the mammalian Sel-10 homolog. J. Biol. Chem. 276, 35847-35853. Abstract Article

Parker, J.S., Roe, S.M., and Barford, D. (2004). Crystal structure of a PIWI protein suggests mechanisms for siRNA recognition and slicer activity. EMBO J. Abstract Article

Pepper, A.S., Killian, D.J., and Hubbard, E.J. (2003). Genetic analysis of Caenorhabditis elegans glp-1 mutants suggests receptor interaction or competition. Genetics 163, 115-132. Abstract

Pepper, A.S.-R., Lo, T.-W., Killian, D.J., Hall, D.H., and Hubbard, E.J. A. (2003). The establishment of Caenorhabditis elegans germline pattern is controlled by overlapping proximal and distal somatic gonad signals. Dev. Biol. 259, 336-350. Abstract Article

Petcherski, A.G., and Kimble, J. (2000). LAG-3 is a putative transcriptional activator in the C. elegans Notch pathway. Nature 405, 364-368. Abstract Article

Puoti, A., and Kimble, J. (1999). The Caenorhabditis elegans sex determination gene mog- 1 encodes a member of the DEAH-box protein family. Mol. Cell Biol. 19, 2189-2197. Abstract

Puoti, A., and Kimble, J. (2000). The hermaphrodite sperm/oocyte switch requires the Caenorhabditis elegans homologs of PRP2 and PRP22. Proc. Natl. Acad. Sci. USA 97, 3276-3281. Abstract Article

Qiao, L., Lissemore, J.L., Shu, P., Smardon, A., Gelber, M.B., and Maine, E. M. (1995). Enhancers of glp-1, a gene required for cell-signaling in Caenorhabditis elegans, define a set of genes required for germline development. Genetics 141, 551-569. Abstract

Roussell, D.L., and Bennett, K.L. (1993). glh-1, a germ-line putative RNA helicase from Caenorhabditis, has four zinc fingers. Proc. Natl. Acad. Sci. USA 90, 9300-9304. Abstract

Ryder, S.P., Frater, L.A., Abramovitz, D.L., Goodwin, E.B., and Williamson, J.R. (2004). RNA target specificity of the STAR/GSG domain post-transcriptional regulatory protein GLD-1. Nat. Struct. Mol. Biol. 11, 20-28. Abstract Article

Schwer, B., and Guthrie, C. (1991). PRP16 is an RNA-dependent ATPase that interacts transiently with the spliceosome. Nature 349, 494-499. Abstract Article

Seydoux, G., and Schedl, T. (2001). The germline in C. elegans: origins, proliferation and silencing. Int. Rev. Cytol. 203, 139-185. Abstract

Seydoux, G., Schedl, T., and Greenwald, I. (1990). Cell-cell interactions prevent a potential inductive interaction between soma and germline in C. elegans. Cell 61,939-951. Abstract Article

Shakes, D.C., Sadler, P.L., Schumacher, J.M., Abdolrasulnia, M., and Golden, A. (2003). Developmental defects observed in hypomorphic anaphase-promoting complex mutants are linked to cell cycle abnormalities. Development 130, 1605-1620. Abstract Article 
Smardon, A., Spoerke, J.M., Stacey, S.C., Klein, M.E., Mackin, N., and Maine, E.M. (2000). EGO-1 is related to RNA-directed RNA polymerase and functions in germ- line development and RNA interference in C. elegans [published erratum appears in Curr. Biol. 2000 May 18;10(10):R393-4]. Curr. Biol. 10, 169-178. Abstract Article

Smith, P., Leung-Chiu, W.M., Montgomery, R., Orsborn, A., Kuznicki, K., Gressman-Coberly, E., Mutapcic, L., and Bennett, K. (2002). The GLH proteins, Caenorhabditis elegans P granule components, associate with CSN-5 and KGB-1, proteins necessary for fertility and with ZYX-1, a predicted cytoskeletal protein. Dev. Biol. 251, 333-347. Abstract Article

Song, J.J., Smith, S.K., Hannon, G.J., and Joshua-Tor, L. (2004). Crystal structure of Argonaute and its implications for RISC slicer activity. Science 305, 1434-1437. Abstract Article

Subramaniam, K., and Seydoux, G. (1999). nos-1 and nos-2, two genes related to Drosophila nanos, regulate primordial germ cell development and survival in Caenorhabditis elegans. Development 126, 4861-4871. Abstract

Sulston, J.E., Schierenberg, E., White, J.G., and Thomson, J.N. (1983). The embryonic cell lineage of the nematode Caenorhabditis elegans. Dev. Biol. 100, 64-119. Abstract Article

Sundaram, M., and Greenwald, I. (1993). Suppressors of a lin-12 hypomorph define genes that interact with both lin-12 and glp-1 in Caenorhabditis elegans. Genetics 135, 765-783. Abstract

Tax, F.E., Thomas, J.H., Ferguson, E.L., and Horvitz, H.R. (1997). Identification and characterization of genes that interact with lin-12 in Caenorhabditis elegans. Genetics 2147, 1675-1695. Abstract

Tian, X., Hansen, D., Schedl, T., and Skeath, J.B. (2004). Epsin potentiates Notch pathway activity in Drosophila and C. elegans. Development 131,5807-5815. Abstract Article

Wang, L., Eckmann, C.R., Kadyk, L.C., Wickens, M., and Kimble, J. (2002). A regulatory cytoplasmic poly(A) polymerase in Caenorhabditis elegans. Nature 419, 312-316. Abstract Article

Watt, F.M., and Hogan, B.L. (2000). Out of Eden: stem cells and their niches. Science 287, 1427-1430. Abstract Article

Wen, C., and Greenwald, I. (1999). p24 proteins and quality control of LIN-12 and GLP-1 trafficking in Caenorhabditis elegans. J. Cell. Biol. 145, 1165-1175. Abstract Article

Wen, C., Metzstein, M.M., and Greenwald, I. (1997). SUP-17, a Caenorhabditis elegans ADAM protein related to Drosophila KUZBANIAN and its role in LIN-12/NOTCH signalling. Development 124, 4759-4767. Abstract

Wickens, M., Bernstein, D.S., Kimble, J., and Parker, R. (2002). A PUF family portrait: 3'UTR regulation as a way of life. Trends Genet. 18, 150-157. Abstract Article

Wilkinson, H.A., Fitzgerald, K., and Greenwald, I. (1994). Reciprocal changes in expression of the receptor lin-12 and its ligand lag-2 prior to commitment in a C. elegans cell fate decision. Cell 79, 1187-1198. Abstract Article

Wu, G., Hubbard, E.J. A., Kitajewski, J.K., and Greenwald, I. (1998). Evidence for functional and physical association between Caenorhabditis elegans SEL-10, a Cdc4p-related protein and SEL-12 presenilin. Proc. Natl. Acad. Sci. USA 95, 15787-15791. Abstract Article

Zhang, B., Gallegos, M., Puoti, A., Durkin, E., Fields, S., Kimble, J., and Wickens, M.P. (1997). A conserved RNA-binding protein that regulates sexual fates in the C. elegans hermaphrodite germ line. Nature 390, 477-484. Abstract Article

Zhong, W., Feng, H., Santiago, F.E., and Kipreos, E.T. (2003). CUL-4 ubiquitin ligase maintains genome stability by restraining DNA-replication licensing. Nature 423, 885-889. Abstract Article

All WormBook content, except where otherwise noted, is licensed under a Creative SOMIEREIGHISRESERVED Commons Attribution License. 